\title{
Numerical Solutions for the Three-Point Boundary Value Problem of Nonlinear Fractional Differential Equations
}

\author{
C. P. Zhang, ${ }^{1}$ J. Niu, ${ }^{2}$ and Y. Z. Lin ${ }^{1}$ \\ ${ }^{1}$ Department of Mathematics, Harbin Institute of Technology, Harbin 150001, China \\ 2 School of Mathematics and Sciences, Harbin Normal University, Harbin 150025, China \\ Correspondence should be addressed to J. Niu, niujing1982@gmail.com
}

Received 22 February 2012; Accepted 30 March 2012

Academic Editor: Svatoslav Staněk

Copyright (C) 2012 C. P. Zhang et al. This is an open access article distributed under the Creative Commons Attribution License, which permits unrestricted use, distribution, and reproduction in any medium, provided the original work is properly cited.

We present an efficient numerical scheme for solving three-point boundary value problems of nonlinear fractional differential equation. The main idea of this method is to establish a favorable reproducing kernel space that satisfies the complex boundary conditions. Based on the properties of the new reproducing kernel space, the approximate solution is obtained by searching least value techniques. Moreover, uniformly convergence and error estimation are provided for our method. Numerical experiments are presented to illustrate the performance of the method and to confirm the theoretical results.

\section{Introduction}

Fractional differential equations have gained considerable importance due to their frequent applications in various fields of science and engineering including physics [1-4], bioengineering [5-7], hydrology [8-10], solid mechanics [11], chaos [12-14], control theory [15], and finance [16-18]. It has been found that fractional derivatives provide an excellent instrument for th e description of memory and hereditary properties of different substances [19]. With these features, the fractional-order models become more practical and realistic than the classical integerd-order models, in which such effects are not taken into account.

Finding exact solutions in closed forms for most differential equations of fractional order is a difficult task. As a result, a number of methods have been proposed and applied successfully to approximate fractional differential equations, such as Adomian decomposition method [20], variational iteration method [21], homotopy analysis method [22], implicit and explicit difference method [23], and collocation method [24]. Especially 
Momani and Odibat have applied He's variational iteration method to fractional differential equations [25-27]. Meanwhile, various fractional order differential equation have been solved very recently including fractional advection-dispersion equations [28, 29], reactiondiffusion system with fractional derivatives [30], fractional partial differential equations fluid mechanics [31], and fractional-order two-point boundary value problem [32].

In contrast to the initial value and two-point boundary value problems, not much attention has been paid to the multipoint fractional boundary value problem (MFBVP). Ahmed and Wang [33] considered existence and uniqueness of solutions for a four-point nonlocal boundary value problem of nonlinear impulsive differential equations of fractional order. Rehman and Khan [34] also studied existence and uniqueness of solutions for a class of multipoint boundary value problems for fractional differential equations. However, the research on the numerical treatment for MFBVP has proceeded very slowly in the recent years. This motivates us to investigate computationally efficient numerical techniques for solving the MFBVP.

In the present work, we are concerned with the numerical solution of the following three-point boundary value problem of fractional differential equation [35] in a reproducing kernel space:

$$
\begin{aligned}
& D_{0^{+}}^{\alpha} u(x)=f(x, u(x)), \quad 0<x<1,3<\alpha \leq 4 \\
& u(0)=u^{\prime}(0)=u^{\prime \prime}(0)=0, \quad u^{\prime \prime}(1)=\beta u^{\prime \prime}(\eta)
\end{aligned}
$$

where $D_{0^{+}}^{\alpha}$ is the Riemann-Liouville fractional derivative and $0<\eta<1$ satisfies $0<\beta \eta^{\alpha-3}<1$, while $f:[0,1] \times[0,+\infty) \rightarrow[0,+\infty)$ is continuous.

Recently, the reproducing kernel space method (RKSM) has been used for obtaining approximate solutions of differential and integral equations [36-38]. However, due to the multipoint boundary value conditions in (1.1), especially for fractional differential equations, it is difficult to find the corresponding reproducing kernel space by applying traditional RKSM.

The aim of this work is to extend the RKSM to derive the numerical solutions of (1.1). One important improvement is that we successfully construct a novel reproducing kernel space so as to overcome difficulties with the nonlocal multipoint boundary value conditions. By using the new reproducing kernel functions, we present an efficient numerical algorithm to solve problem (1.1). The emphasis of the result is that uniformly convergence of the approximate solution, error estimation, and complexity analysis of our algorithm are studied.

The organization of this paper is as follows. In Section 2, we present some important definitions and preparations used in this paper. In Section 3, we construct and develop algorithms for solving nonlinear fractional differential equation with three-point boundary value conditions. In Section 4 the proposed methods are applied to several examples. Also a conclusion is given in Section 5 .

\section{The Construction of a New Reproducing Kernel Space}

We give some basic definitions and theories which are used further in this paper. 
Definition 2.1 (see [39]). The Riemann-Liouville fractional derivative of order $\alpha$ is defined as

$$
D_{0^{+}}^{\alpha} u(x)=\frac{1}{\Gamma(n-s)}\left(\frac{d}{d x}\right)^{n} \int_{0}^{x} \frac{u(t)}{(x-t)^{\alpha-n+1}} d t
$$

$\Gamma(\cdot)$ is the gamma function and $n=[\alpha]+1,[\alpha]$ denotes the integerd part of number $\alpha$.

Definition 2.2 (see [40]). Let $W$ be a Hilbert function space on a set $X$. $W$ is called a reproducing kernel space if and only if for any $x \in X$, there exists a unique function $K_{x}(y) \in W$, such that $\left\langle f, K_{x}\right\rangle=u(x)$ for any $u \in W$. Meanwhile, $K(x, y) \doteq K_{x}(y)$ is called a reproducing kernel.

We now define a new reproducing kernel space $W$ which includes the nonlocal boundary value conditions and give an explicit representation formula for calculation of the reproducing kernel.

Definition 2.3. $W \doteq W[0,1]=\left\{u(x) \mid u^{(4)}(x)\right.$ is an absolutely continuous real value function in $\left.[0,1], u^{(5)}(x) \in L^{2}[0,1], u(0)=u^{\prime}(0)=u^{\prime \prime}(0)=0, u^{\prime \prime}(1)-\beta u^{\prime \prime}(\eta)=0,0<\eta<1,0<\beta \eta^{\alpha-3}<1\right\}$.

The inner product is given by

$$
\langle u(x), v(x)\rangle=u^{\prime \prime}(\eta) v^{\prime \prime}(\eta)+u^{(4)}(0) v^{(4)}(0)+\int_{0}^{1} u^{(5)}(x) v^{(5)}(x) d x .
$$

Theorem 2.4. $W$ is a reproducing kernel space, that is, there exists a function $K(x, y) \in W$, for any fixed $y \in[0,1]$ and any $u(x) \in W$, such that $u(y)=\langle u(x), K(x, y)\rangle$. Moreover, the reproducing kernel can be denoted by

$$
K(x, y)= \begin{cases}k_{1}(x, y)=a_{1}+a_{2} x+a_{3} x^{2}+\cdots+a_{10} x^{9}, & y<x<\eta, \\ k_{2}(x, y)=b_{1}+b_{2} x+b_{3} x^{2}+\cdots+b_{10} x^{9}, & y<\eta<x, \\ k_{3}(x, y)=c_{1}+c_{2} x+c_{3} x^{2}+\cdots+c_{10} x^{9}, & \eta<y<x, \\ k_{4}(x, y)=d_{1}+d_{2} x+d_{3} x^{2}+\cdots+d_{10} x^{9}, & \eta<x<y, \\ k_{5}(x, y)=e_{1}+e_{2} x+e_{3} x^{2}+\cdots+e_{10} x^{9}, & x<\eta<y, \\ k_{6}(x, y)=f_{1}+f_{2} x+f_{3} x^{2}+\cdots+f_{10} x^{9}, & x<y<\eta .\end{cases}
$$

Proof. For any $u(x) \in W$, we only need to prove that there exists a $K(x, y) \in W$ for any fixed $y \in[0,1]$ and any $u(x) \in W, K(x, y)$ must satisfy

$$
\langle u(x), K(x, y)\rangle=u(y)
$$


By (2.2), we get

$$
\begin{aligned}
\langle u(x), K(x, y)\rangle= & \left.u^{\prime \prime}(\eta) \frac{\partial^{2} K(x, y)}{\partial x^{2}}\right|_{x=\eta}+\left.u^{(4)}(0) \frac{\partial^{4} K(x, y)}{\partial x^{4}}\right|_{x=0} \\
& +\int_{0}^{1} u^{(5)}(x) \frac{\partial^{5} K(x, y)}{\partial x^{5}} d x
\end{aligned}
$$

We have the following equality using the integration by parts:

$$
\int_{0}^{1} u^{(5)}(x) \frac{\partial^{5} K(x, y)}{\partial x^{5}} d x=\left.\sum_{i=0}^{4}(-1)^{4-i} u^{(i)}(x) \frac{\partial^{9-i} K(x, y)}{\partial x^{9-i}}\right|_{0} ^{1}-\int_{0}^{1} u(x) \frac{\partial^{10} K(x, y)}{\partial x^{10}} d x
$$

Substituting (2.6) in (2.5), we get

$$
\begin{aligned}
\langle u(x), K(x, y)\rangle= & u^{\prime \prime}(\eta)\left(\left.\frac{\partial^{2} K(x, y)}{\partial x^{2}}\right|_{x=\eta}-\left.\beta \frac{\partial^{7} K(x, y)}{\partial x^{7}}\right|_{x=1}\right) \\
& +u^{(4)}(0)\left(\left.\frac{\partial^{4} K(x, y)}{\partial x^{4}}\right|_{x=0}-\left.\frac{\partial^{5} K(x, y)}{\partial x^{5}}\right|_{x=0}\right) \\
& +\left.u^{(3)}(0) \frac{\partial^{6} K(x, y)}{\partial x^{6}}\right|_{x=0}+\left.\sum_{i=0}^{1}(-1)^{4-i} u^{(i)}(1) \frac{\partial^{9-i} K(x, y)}{\partial x^{9-i}}\right|_{x=1} \\
& +\left.\sum_{i=3}^{4}(-1)^{4-i} u^{(i)}(1) \frac{\partial^{9-i} K(x, y)}{\partial x^{9-i}}\right|_{x=1}-\int_{0}^{1} u(x) \frac{\partial^{10} K(x, y)}{\partial x^{10}} d x .
\end{aligned}
$$

Therefore, by (2.4) $K(x, y)$ is the solution of the following generalized differential equation:

$$
\begin{gathered}
\left.\frac{\partial^{2} K(x, y)}{\partial x^{2}}\right|_{x=\eta}-\left.\beta \frac{\partial^{7} K(x, y)}{\partial x^{7}}\right|_{x=1}=0 \\
\left.\frac{\partial^{4} K(x, y)}{\partial x^{4}}\right|_{x=0}-\left.\frac{\partial^{5} K(x, y)}{\partial x^{5}}\right|_{x=0}=0 \\
\left.\frac{\partial^{6} K(x, y)}{\partial x^{6}}\right|_{x=0}=0 \\
\left.\frac{\partial^{9-i} K(x, y)}{\partial x^{9-i}}\right|_{x=1}=0, \quad i=0,1,3,4 \\
-\frac{\partial^{10} K(x, y)}{\partial x^{10}}=\delta(x-y)
\end{gathered}
$$


where $\delta$ denotes $\delta$ function. For $x \neq y$, it is known that $K(x, y)$ is the solution of the following linear homogeneous differential equation:

$$
\frac{\partial^{10} K(x, y)}{\partial x^{10}}=0
$$

with the boundary value conditions:

$$
\begin{gathered}
\left.\frac{\partial^{2} K(x, y)}{\partial x^{2}}\right|_{x=\eta}-\left.\beta \frac{\partial^{7} K(x, y)}{\partial x^{7}}\right|_{x=1}=0 \\
\left.\frac{\partial^{4} K(x, y)}{\partial x^{4}}\right|_{x=0}-\left.\frac{\partial^{5} K(x, y)}{\partial x^{5}}\right|_{x=0}=0 \\
\left.\frac{\partial^{6} K(x, y)}{\partial x^{6}}\right|_{x=0}=0 \\
\left.\frac{\partial^{9-i} K(x, y)}{\partial x^{9-i}}\right|_{x=1}=0, \quad i=0,1,3,4
\end{gathered}
$$

We find that (2.9) has characteristic equation $\lambda^{10}=0$, and the eigenvalue $\lambda=0$ is a root whose multiplicity is 10. Applying the feature of functions in $W$, the general solution of (2.9) is given by the universal representation as (2.3), in which every function $k_{i}(x, y)(i=1,2,3, \ldots, 6)$ has the situation in Figure 1. Next we will calculate 60 coefficients in (2.3). to $x$, we have

By integrating repeatedly $\partial^{10} K(x, y) / \partial t^{10}=-\delta(x-y)$ from $y-\varepsilon$ to $y+\varepsilon$ with respect

$$
\left.\frac{\partial^{9} K(x, y)}{\partial x^{9}}\right|_{x=y-\varepsilon} ^{y+\varepsilon}=-1,\left.\quad \frac{\partial^{i} K(x, y)}{\partial x^{i}}\right|_{x=y-\varepsilon} ^{y+\varepsilon}=0, \quad i=8,7,6, \ldots, 0,
$$

and the following 20 equations are inferred as $\varepsilon \rightarrow 0$

$$
\begin{gathered}
\left.\frac{\partial^{9} k_{1}(x, y)}{\partial x^{9}}\right|_{x=y}-\left.\frac{\partial^{9} k_{6}(x, y)}{\partial x^{9}}\right|_{x=y}=-1, \\
\left.\frac{\partial^{9} k_{3}(x, y)}{\partial x^{9}}\right|_{x=y}-\left.\frac{\partial^{9} k_{4}(x, y)}{\partial x^{9}}\right|_{x=y}=-1, \\
\left.\frac{\partial^{i} k_{1}(x, y)}{\partial x^{i}}\right|_{x=y}-\left.\frac{\partial^{i} k_{6}(x, y)}{\partial x^{i}}\right|_{x=y}=0, \quad i=8,7,6, \ldots, 0, \\
\left.\frac{\partial^{i} k_{3}(x, y)}{\partial x^{i}}\right|_{x=y}-\left.\frac{\partial^{i} k_{4}(x, y)}{\partial x^{i}}\right|_{x=y}=0, \quad i=8,7,6, \ldots, 0 .
\end{gathered}
$$




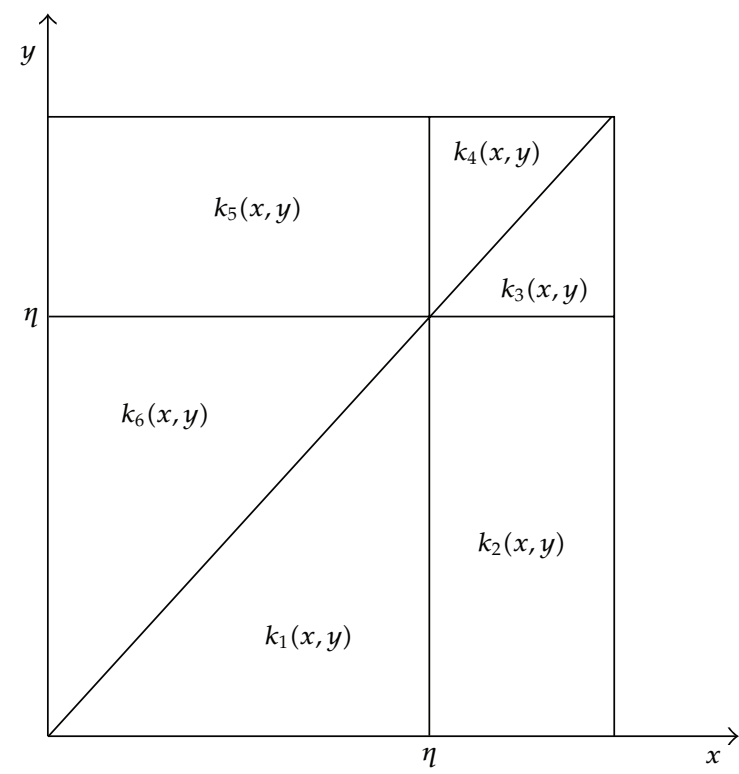

Figure 1: Plot of distribution for $k_{i}(x, y), i=1,2, \ldots, 6$.

By (2.10), one can obtain 14 equations. In view of boundary value conditions, the following 8 equations can be obtained:

$$
\begin{aligned}
\left.\frac{\partial^{i} k_{5}(x, y)}{\partial x^{i}}\right|_{x=0}=0 \quad(i=0,1,2), & \left.\frac{\partial^{i} k_{6}(x, y)}{\partial x^{i}}\right|_{x=0}=0 \quad(i=0,1,2), \\
\left.\frac{\partial^{2} k_{2}(x, y)}{\partial x^{2}}\right|_{x=1}-\left.\beta \frac{\partial^{2} k_{2}(x, y)}{\partial x^{i}}\right|_{x=\eta}=0, & \left.\frac{\partial^{2} k_{3}(x, y)}{\partial x^{2}}\right|_{x=1}-\left.\beta \frac{\partial^{2} k_{4}(x, y)}{\partial x^{i}}\right|_{x=\eta}=0 .
\end{aligned}
$$

Finally, due to the smoothing of functions in $W$, we get 18 equations for $i=0,1,2, \ldots, 8$

$$
\begin{aligned}
& \left.\frac{\partial^{i} k_{1}(x, y)}{\partial x^{i}}\right|_{x=\eta}-\left.\frac{\partial^{i} k_{2}(x, y)}{\partial x^{i}}\right|_{x=\eta}=0, \\
& \left.\frac{\partial^{i} k_{4}(x, y)}{\partial x^{i}}\right|_{x=\eta}-\left.\frac{\partial^{i} k_{5}(x, y)}{\partial x^{i}}\right|_{x=\eta}=0 .
\end{aligned}
$$

Hence, the unknown coefficients of $K(x, y)$ are governed by solving the 60 independent equations by (2.10)-(2.14). 


\section{Description of the Proposed Numerical Method}

The method consists of two steps. In the first step, a normal orthogonal basis is established in the reproducing kernel space $W$, and in the second step, it is used to successively obtain the approximate solution of (1.1). Let us consider these steps in detail.

\subsection{A Normal Orthogonal Basis in $W$}

Define a bounded linear operator $\mathrm{T}: W[0,1] \rightarrow L^{1}[0,1]$ satisfying

$$
\mathrm{T} u(x)=D_{0^{+}}^{\alpha} u(x)
$$

The proof of existence and uniqueness of solution for (1.1) has been studied in [35]. Therefore T is reversible. Now, (1.1) is turned into the following operator equation in $W$ :

$$
\mathrm{T} u(x)=f(x, u(x))
$$

Choosing a countable dense subset $\left\{x_{i}\right\}_{i=1}^{\infty}$ on $[0,1]$, for the reproducing kernel $K(x, y)$ of $W$, we define a complete system in $W$ as

$$
\psi_{i}(x)=(\mathrm{T} K(x,(\cdot)))\left(x_{i}\right)=\frac{1}{\Gamma(4-\alpha)} \frac{\partial^{4}}{\partial x_{i}^{4}} \int_{0}^{x_{i}} \frac{K(x, y)}{\left(x_{i}-y\right)^{\alpha-3}} d y
$$

Then the orthogonal system of $W$ is derived from Gram-Schmidt orthogonalization process, namely,

$$
\widetilde{\psi}_{i}(x)=\sum_{k=1}^{i} \beta_{i k} \psi_{k}(x)
$$

Next, the complexity estimation of the orthogonal basis is discussed. We know that the orthogonal basis is obtained by orthogonalization of complete system $\psi_{i}(x)$. The algorithm with time complexity may be analyzed as following.

Step 1 (Computing $\left\langle\psi_{i}(x), \psi_{k}(x)\right\rangle$ ). In fact, according to the properties of reproducing kernel and bounded linear operator, we have

$$
\left\langle\psi_{i}(x), \psi_{k}(x)\right\rangle=\mathrm{T} \psi_{i}\left(x_{k}\right)
$$

Thus, we only need to calculate the specific function value $\mathrm{T} \psi_{i}\left(x_{k}\right)$ instead of the usual integral. Denote the computing time of the specific function value by $T$. It needs $(((n) n+$ 1))) /2) $T$ to compute those $\left\langle\psi_{i}(x), \psi_{k}(x)\right\rangle k=1,2, \ldots, i, i=1,2, \ldots, n$. 
Step 2. Orthogonalization can be obtained by the following cycle.

Firstly, let $\beta_{11}=1 /\left\|\psi_{1}\right\|$ and

$$
C_{i j}=\left\langle\psi_{i}, \tilde{\psi}_{j}\right\rangle_{H}=\left\langle\psi_{i}, \sum_{k=1}^{i} \beta_{j k} \psi_{k}\right\rangle=\sum_{k=1}^{i} \beta_{j k}\left\langle\psi_{i}, \psi_{k}\right\rangle .
$$

For $1 \leq j \leq i-1$, it takes $(i(i-1)) / 2$ times multiplication operations for computing $C_{i j}$. Secondly, we denote

$$
\beta_{i m}=\frac{-\sum_{k=m}^{i-1} C_{i k} \beta_{k m}}{\left\|\widetilde{\psi}_{i}\right\|}
$$

where

$$
\left\|\tilde{\psi}_{i}\right\|=\left(\left\|\psi_{i}\right\|^{2}-\sum_{k=1}^{i-1}\left|C_{i k}\right|^{2}\right)^{1 / 2}=\left(\left\langle\psi_{i}, \psi_{i}\right\rangle-\sum_{k=1}^{i-1} C_{i k}^{2}\right)^{1 / 2}
$$

For $1 \leq m \leq i-1,((i+2)(i-1)) / 2$ times multiplication operations are demanded to compute $\beta_{i m}$. Finally, for $2 \leq i \leq n$, it uses all $(n(n-1)(2 n+3)) / 4$ times multiplication operations. To sum up, from Step 1 and Step 2, we get orthogonal basis complexity result of $(n(n-1)(2 n+$ 3)) $/ 4$ times multiplication operations plus $((n(n+1)) / 2) T$. Therefore, the construction of orthogonal basis costs a total of $\mathcal{O}\left(n^{3}\right)$ operations.

\subsection{The Approximate Solution of (3.2) in $W$}

Theorem 3.1. If $u(x)$ is the solution of (3.2), then

$$
u(x)=\sum_{i=1}^{\infty} \sum_{k=1}^{i} \beta_{i k} f\left(x_{k}, u\left(x_{k}\right)\right) \tilde{\psi}_{i}(x)
$$

Proof. According to the orthogonal basis $\left\{\tilde{\psi}_{i}(x)\right\}_{i=1}^{\infty}$ of $W$, we have

$$
\begin{aligned}
u(x) & =\sum_{i=1}^{\infty}\left\langle u(\cdot), \widetilde{\psi}_{i}(x)\right\rangle \widetilde{\psi}_{i}(x)=\sum_{i=1}^{\infty} \sum_{k=1}^{i} \beta_{i k}\left\langle u(\cdot), \psi_{k}(x)\right\rangle \widetilde{\psi}_{i}(x) \\
& =\sum_{i=1}^{\infty} \sum_{k=1}^{i} \beta_{i k}\left\langle u(\cdot),\left(\mathrm{T} K\left(x_{k}, \cdot\right)\right)\right\rangle \widetilde{\psi}_{i}(x)=\sum_{i=1}^{\infty} \sum_{k=1}^{i} \beta_{i k}\left(\mathrm{~T}\left\langle u(\cdot), K\left(x_{k}, \cdot\right)\right\rangle\right) \widetilde{\psi}_{i}(x) \\
& =\sum_{i=1}^{\infty} \sum_{k=1}^{i} \beta_{i k}(\mathrm{~T} u)\left(x_{k}\right) \tilde{\psi}_{i}(x)=\sum_{i=1}^{\infty} \sum_{k=1}^{i} \beta_{i k} f\left(x_{k}, u\left(x_{k}\right)\right) \widetilde{\psi}_{i}(x) .
\end{aligned}
$$


Denoting $\alpha_{k}=f\left(x_{k}, u\left(x_{k}\right)\right)$, (3.9) can be rewritten as

$$
u(x)=\sum_{i=1}^{\infty} \sum_{k=1}^{i} \beta_{i k} \alpha_{k} \tilde{\psi}_{i}(x)
$$

To obtain $\alpha_{k}$, we truncate the series of the left-hand side of (3.11)

$$
u_{n}(x)=\sum_{i=1}^{n} \sum_{k=1}^{i} \beta_{i k} \alpha_{k} \widetilde{\psi}_{i}(x)
$$

Then we get $\alpha_{k}$ based on the minimum point of function

$$
J\left(\alpha_{1}, \alpha_{2}, \ldots, \alpha_{n}\right)=\sum_{k=1}^{n}\left[f\left(x_{k}, u_{n}\left(x_{k}\right)\right)-\alpha_{k}\right]^{2}
$$

Consequently, the approximate solution of (3.2) can be obtained.

Lemma 3.2. The approximate solution $u_{n}(x)$ and its derivatives $u_{n}^{(i)}(x),(i=1,2,3,4)$ uniformly converge to exact solution $u(x)$ and its derivatives, respectively.

Proof. For any $x \in[0,1]$, according to the boundedness of $\left\|\partial_{x}^{i} K(x, y)\right\|(i=0,1,2,3,4)$ and reproducing property of $K(x, y)$, the following conclusion is obtained as $n \rightarrow \infty$

$$
\begin{aligned}
\left|u_{n}^{(i)}(x)-u^{(i)}(x)\right| & =\left|\left(u_{n}(x)-u(x)\right)^{(i)}\right|=\left|\partial_{x}^{i}\left\langle u_{n}(y)-u(y), K(x, y)\right\rangle\right| \\
& =\left|\left\langle u_{n}(y)-u(y), \partial_{x}^{i} K(x, y)\right\rangle\right| \\
& \leq\left\|u_{n}-u\right\|\left\|\partial_{x}^{i} K(x, y)\right\| \leq M\left\|u_{n}-u\right\| \rightarrow 0 .
\end{aligned}
$$

Finally, we give the process to obtain $\alpha_{k}$ :

$\left(1^{\circ}\right)$ pick any initial value $\alpha_{k}^{0}$;

$\left(2^{\circ}\right)$ substitute $\alpha_{k}^{0}$ into (3.12) and get $u_{n}(x)$;

$\left(3^{\circ}\right)$ substitute $\alpha_{k}^{0}, u_{n}(x)$ into (3.13) and compute the value $J\left(\alpha_{1}^{0}, \alpha_{2}^{0}, \ldots, \alpha_{n}^{0}\right)$;

$\left(4^{\circ}\right)$ if $J\left(\alpha_{1}^{0}, \alpha_{2}^{0}, \ldots, \alpha_{n}^{0}\right)<10^{-20}$ then the computations terminate, otherwise substituting $u_{n}(x)$ into (3.13) yields a new minimum point $\alpha_{k}^{1}$;

$\left(5^{\circ}\right)$ calculate $J\left(\alpha_{1}^{1}, \alpha_{2}^{1}, \ldots, \alpha_{n}^{1}\right)$;

$\left(6^{\circ}\right)$ if $J\left(\alpha_{1}^{1}, \alpha_{2}^{1}, \ldots, \alpha_{n}^{1}\right)<J\left(\alpha_{1}^{0}, \alpha_{2}^{0}, \ldots, \alpha_{n}^{0}\right)$ then replace $\alpha_{k}^{0}$ with $\alpha_{k}^{1}$ and return to $2^{\circ}$, otherwise give up $\alpha_{k}^{1}$ and return to $1^{\circ}$ and pick another initial value. 
Theorem 3.3. $\left|u_{n}(x)-u(x)\right|=o(1 / n)$.

Proof. Firstly, because of denseness, for any $x \in[0,1]$ and $n \in N$, we take $x_{i} \in\left\{x_{1}, x_{2}, \ldots\right\}$, $i \leq n$ such that $\left|x-x_{i}\right|<1 / n$. Then due to the reproducing property and the property of projector $P_{n}$, it follows that

$$
\begin{aligned}
\mathrm{T} u_{n}\left(x_{i}\right) & =\left\langle u_{n}(\cdot), \mathrm{TK}\left(x_{i}, \cdot\right)\right\rangle=\left\langle P_{n} u(\cdot), \psi_{i}(\cdot)\right\rangle=\left\langle u(\cdot), P_{n} \psi_{i}(\cdot)\right\rangle \\
& =\left\langle u(\cdot), \psi_{i}(\cdot)\right\rangle=\mathrm{T}\left\langle u(\cdot), K\left(x_{i}, \cdot\right)\right\rangle=\mathrm{T} u\left(x_{i}\right) .
\end{aligned}
$$

This implies that

$$
\begin{aligned}
\mathrm{T} u_{n}(x)-\mathrm{T} u(x) & =\mathrm{T} u_{n}(x)-\mathrm{T} u_{n}\left(x_{i}\right)-\left(\mathrm{T} u(x)-\mathrm{T} u\left(x_{i}\right)\right) \\
& =\left\langle u_{n}(\cdot), \mathrm{T} K(x, \cdot)-\mathrm{T} K\left(x_{i}, \cdot\right)\right\rangle-\left\langle u(\cdot), \mathrm{T} K(x, \cdot)-\mathrm{T} K\left(x_{i}, \cdot\right)\right\rangle \\
& =\left\langle u_{n}(\cdot)-u(\cdot), \mathrm{T} K(x, \cdot)-\mathrm{T} K\left(x_{i}, \cdot\right)\right\rangle .
\end{aligned}
$$

By the mean value theorem, we have

$$
\mathrm{T} K(x, \cdot)-\mathrm{T} K\left(x_{i}, \cdot\right)=\left(x-x_{i}\right) \frac{\partial}{\partial y} \mathrm{~T} K(y, \cdot)
$$

Finally, the following conclusion follows from above:

$$
\begin{aligned}
\left|u_{n}(x)-u(x)\right| & =\left\langle u_{n}-u, \mathrm{~T}^{-1}\left(\mathrm{~T} K(x, \cdot)-\mathrm{T} K\left(x_{i}, \cdot\right)\right)\right\rangle \\
& \leq\left\|\mathrm{T}^{-1}\right\|\left\|u_{n}-u\right\|\left\|\mathrm{T} K(x, \cdot)-\mathrm{T} K\left(x_{i}, \cdot\right)\right\| \\
& \leq\left\|\mathrm{T}^{-1}\right\|\left\|u_{n}-u\right\|\left|x-x_{i}\right|\left\|\frac{\partial}{\partial y} \mathrm{~T} K(y, \cdot)\right\| .
\end{aligned}
$$

Thus, according to $\left\|u_{n}-u\right\| \rightarrow 0,\left|x-x_{i}\right|<1 / n$ and the boundedness of $\left\|(\partial / \partial y) \mathrm{TK}\left(y_{1} \cdot\right)\right\|$, we get $\left|u_{n}(x)-u(x)\right|=o(1 / n)$.

\section{Numerical Experiments}

In this section, we give some computational results of three numerical experiments with methods based on preceding sections, to support our theoretical discussion.

Example 4.1. Consider the following three-point boundary value problem of nonlinear fractional differential equation [35]:

$$
\begin{array}{cl}
D_{0^{+}}^{7 / 2} u(x)=f(x, u(x)), & 0<x<1, \\
u(0)=u^{\prime}(0)=u^{\prime \prime}(0)=0, & u^{\prime \prime}(1)=u^{\prime \prime}\left(\frac{1}{4}\right),
\end{array}
$$


with an exact solution $u(x)=\left(x^{3} / 100\right)\left(425+170 x-128 x^{3}\right)$. Here $f(x, u(x))=\ln (2+$ $\left.\left(\left(17 x^{3}\right) / 4\right)+\left(\left(17 x^{4}\right) / 10\right)+\left(\left(32 x^{6}\right) / 25\right)\right)+\left(1275+4080 x-24576 x^{3}\right) / 50 \sqrt{x} \Gamma(0.5)-\ln (2+u(x))$.

For this example, we solved the three-point boundary value problem, by applying the technique described in preceding section as following.

Step 1. By using the representation formula for calculation of reproducing kernel in Section 2, the concrete expression of $K(x, y)$ for the three-point boundary value conditions in (4.1) is given as

$$
\begin{aligned}
& k_{1}(x, y)=\frac{y^{3}}{5095276462080} \\
& \times\left(-21 x^{3}(-3141613209+64 x(16138815+x(3227763+4 x(-219394+3007 x))))\right. \\
& +20160 x^{3}\left(-1075921+4 x\left(108017-336 x+64 x^{3}\right)\right) y \\
& +4032 x^{3}\left(-1075921+4 x\left(108017-336 x+64 x^{3}\right)\right) y^{2} \\
& -768 x^{2}\left(-658182+x\left(21049+64 x\left(-105-21 x+4 x^{3}\right)\right)\right) y^{4} \\
& \left.-126370944 x y^{5}+14041216 y^{6}\right) ; \\
& k_{2}(x, y)=\frac{y^{3}}{81524423393280} \\
& \times(105(1889+64 y)+4(-21 x(66115+4 x(-198345 \\
& +x(-3140290909+16 x(64224685+652592 x(21+(-7+x) x))))) \\
& -6720 x(7+4 x(-21+x(3227903+4 x(-324191+64 x(21+(-7+x) x))))) y \\
& -336(-1+4 x(7+4 x(-21 \\
& +x(3227903+4 x(-324191+64 x(21+(-7+x) x)))))) y^{2} \\
& +64(-1+4 x(7+4 x(1974525 \\
& +x(-63007+16 x(1225+16 x(21+(-7+x) x)))))) y^{4} \\
& \left.\left.-505483 x y^{5}+56164 y^{6}\right)\right) \text {; } \\
& k_{3}(x, y)=\frac{1}{3913172322877440} \\
& \times((-1+28 y+4(x(7+4 x(-21+x(595175+16 x(1225+16 x(21+(-7+x) x))))) \\
& -28 x(7+4 x(-21+x(595175+16 x(1225+16 x(21+(-7+x) x))))) y \\
& +84(-1+4 x(7+4 x(-21+x(59575+16 x(1225+16 x(21+(-7+x) x)))))) y^{2} \\
& -28(-85025+4 x(595175+4 x(-1785525
\end{aligned}
$$




$$
\begin{gathered}
+x(-28250714681+16 x(5780465+5873648 x(21+(-7+x) x)))))) y^{3} \\
-15680(-5+4 x(35+4 x(-105 \\
+x(16515619+256 x(-26062+5 x(21+(-7+x) x)))))) y^{4} \\
-21504\left(-1+4 x\left(7+4 x\left(-21+x\left(25697 y^{5}\right.\right.\right.\right. \\
\left.\left.\left.\left.\left.\left.\left.+516677 y^{6}+16 x(1225+(-7+x) x) y^{7}-242632 x y^{8}+269591 y^{9}\right)\right)\right)\right)\right)\right)\right) ; \\
k_{4}(x, y)=k_{3}(y, x) ; \quad k_{5}(x, y)=k_{2}(y, x) ; \quad k_{6}(x, y)=k_{1}(y, x) .
\end{gathered}
$$

Step 2. According to the numerical algorithm in Section 3, we get

$$
\psi_{i}(x)=\frac{1}{\Gamma(0.5)} \frac{\partial^{4}}{\partial x_{i}^{4}} \int_{0}^{x_{i}} \frac{K(x, y)}{\sqrt{x_{i}-y}} d y
$$

By (3.4) we get the orthogonalization coefficients $\beta_{i k}$ and $\widetilde{\psi}_{i}(x)$. Then the approximate solution $u_{n}(x)$ can be obtained iteratively by (3.12).

The obtained numerical results are displayed in Table 1. Furthermore, the graph of $u(x)$ and $u_{n}(x)$ for $n=128$ is plotted in Figure 2. It can be shown that the numerical solutions agree with exact solution by means of the proposed method.

Due to the multipoint boundary value conditions, to our knowledge, there is no the same example as (1.1) in the literature about numerical method. For the purpose of comparison, we compare the approximate solution of our method, together with the approximate solution by Adomian Decomposition (AD) method given in [41].

Example 4.2. In this example, we consider the following nonlinear fourth-order fractional integrodifferential equation of the form:

$$
\begin{gathered}
D_{0^{+}}^{\alpha} y(x)=1+\int_{0}^{x} e^{-t} y^{2}(t) d t, \quad 0<x<1,3<\alpha \leq 4, \\
y(0)=y^{\prime \prime}(0)=1, \quad y(1)=y^{\prime \prime}(1)=e .
\end{gathered}
$$

In this case, a similar numerical method can be proposed as (1.1). In brief, the conditions $u(0)=u^{\prime}(0)=u^{\prime \prime}(0)=0, u^{\prime \prime}(1)=\beta u^{\prime \prime}(\eta)$ in the reproducing kernel space $W$ are replaced by $y(0)=y^{\prime \prime}(0)=1, y(1)=y^{\prime \prime}(1)=e$. There are some slight changes in the process of derivation. The obtained numerical results for $\alpha=3.25$ are displayed in Table 2 . 
Table 1: Numerical results for $n=128$ for Example 4.1.

\begin{tabular}{lccc}
\hline Node & Exact solution & Approximate solution & Absolute error \\
\hline $1 / 8$ & 0.00871094 & 0.0087107 & $2.39103 e-07$ \\
$1 / 4$ & 0.0727344 & 0.0727329 & $1.46440 e-06$ \\
$3 / 8$ & 0.25418 & 0.254176 & $4.14403 e-06$ \\
$1 / 2$ & 0.61750 & 0.617491 & $8.61550 e-06$ \\
$5 / 8$ & 1.22070 & 1.22069 & $1.51075 e-05$ \\
$3 / 4$ & 2.10305 & 2.10302 & $2.36936 e-05$ \\
$7 / 8$ & 3.26922 & 3.26918 & $3.42573 e-05$ \\
1 & 4.67000 & 4.66995 & $4.65042 e-05$ \\
\hline
\end{tabular}
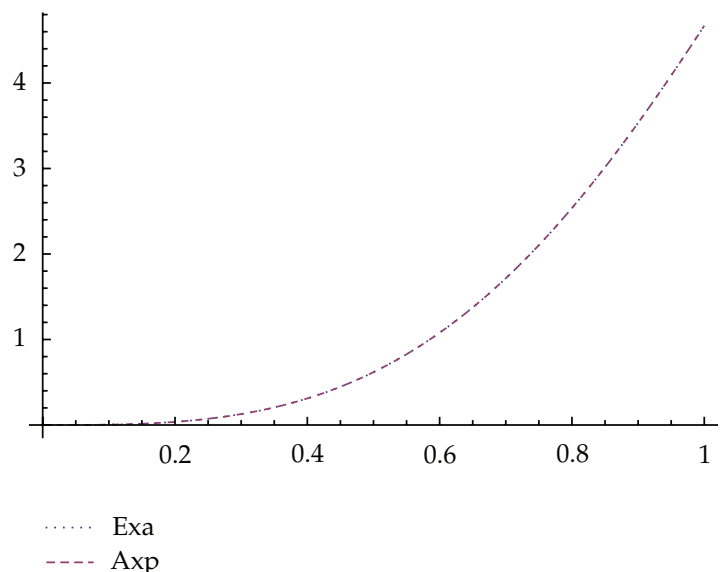

Figure 2: Comparison of the approximate solution $u_{n}(x)$ and $u(x)$ at $n=128$ for Example 4.1.

Table 2: The approximate solution in our method and in [41] for Example 4.2.

\begin{tabular}{lcc}
\hline Node & Present method & AD method in [41] \\
\hline 0.1 & 1.12257 & 1.1202485492579 \\
0.2 & 1.27641 & 1.2624009459051 \\
0.3 & 1.42190 & 1.4293559759369 \\
0.4 & 1.64819 & 1.6248578688995 \\
0.5 & 1.90926 & 1.8534779619798 \\
0.6 & 2.18762 & 2.1206550639506 \\
0.7 & 2.50183 & 2.4327620675062 \\
0.8 & 2.83318 & 2.7971904644190 \\
0.9 & 3.29015 & 3.2224499000323 \\
\hline
\end{tabular}

Example 4.3. Consider the nonlinear fifth-order fractional differential equation with threepoint boundary value conditions as following:

$$
\begin{gathered}
D_{0^{+}}^{9 / 2} u(x)=\sin \left(19 x^{5}-(760 / 93) x^{7}\right)+\frac{1520 \sqrt{x}\left(93-448 x^{2}\right)}{31 \Gamma(0.5)}-\sin u(x), \quad 0<x<1, \\
u(0)=u^{\prime}(0)=u^{\prime \prime}(0)=u^{\prime \prime \prime}(0)=0, \quad u^{\prime \prime}(1)=u^{\prime \prime}\left(\frac{1}{2}\right) .
\end{gathered}
$$


Table 3: Numerical results for $n=128$ for Example 4.3.

\begin{tabular}{lccc}
\hline Node & Exact solution & Approximate solution & Absolute error \\
\hline $1 / 8$ & 0.00533087 & 0.00533148 & $6.11643 e-08$ \\
$1 / 4$ & 0.0194715 & 0.0194721 & $6.18588 e-07$ \\
$3 / 8$ & 0.135717 & 0.135719 & $2.27009 e-06$ \\
$1 / 2$ & 0.529906 & 0.529911 & $4.71005 e-06$ \\
$5 / 8$ & 1.48634 & 1.48635 & $9.38315 e-06$ \\
$3 / 4$ & 3.34208 & 3.3421 & $1.43306 e-05$ \\
$7 / 8$ & 6.59401 & 6.59403 & $2.51263 e-05$ \\
1 & 10.8280 & 10.8280 & $5.46427 e-05$
\end{tabular}

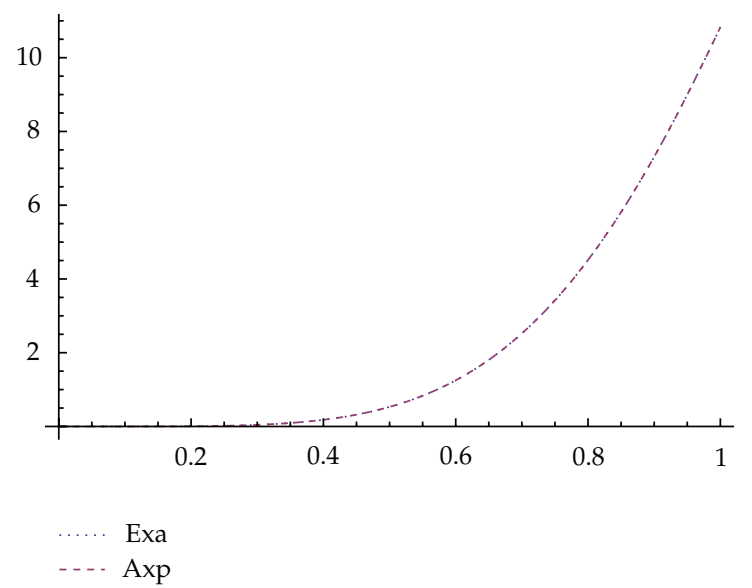

Figure 3: Comparison of the approximate solution $u_{n}(x)$ and $u(x)$ at $n=128$ for Example 4.3.

According to the numerical scheme above, one can obtain the approximation $u_{n}(x)$ of $u(x)$ for $n=128$. The numerical results are displayed in Table 3 and Figure 3, which show that the numerical solutions agree with exact solution.

\section{Conclusion}

In this paper, the RKSM is applied to derive approximate analytical solution of nonlinear fractional-order differential equations with three-point boundary value conditions. We have constructed a novel reproducing kernel space and give the way to express the reproducing kernel function, while traditional RKSM still can not be mentioned. The explicit series solution is obtained using the orthogonal basis established in the new reproducing kernel space. The numerical results given in the previous section demonstrate the better accuracy of our algorithms. Moreover, the numerical algorithms introduced in this paper can be well suited for handling general linear and nonlinear fractional-order differential equations with multipoint boundary conditions. We note that the corresponding analytical and numerical solutions are obtained using Mathematica 7.0. 


\section{Acknowledments}

This paper was supported by Youth Foundation of Heilongjiang Province under grant QC2010036 and also supported by Fundamental Research Funds for the Central Universities under Grant no.HIT.NSRIF.2009050 and Academic Foundation for Youth of Harbin Normal University 11KXQ-04 and 10KXQ-05.

\section{References}

[1] G. Zaslavsky, Hamiltonian Chaos and Fractional Dynamics, Oxford University Press, Oxford, UK, 2008.

[2] B. West, M. Bologna, and P. Grigolini, Physics of Fractal Operators, Springer, New York, NY, USA, 2003.

[3] M. M. Meerschaert and H. P. Scheffler, "Semistable Lévy motion," Fractional Calculus \& Applied Analysis, vol. 5, no. 1, pp. 27-54, 2002.

[4] M. M. Meerschaert, D. Benson, and B. Baeumer, "Operator Levy motion and multiscaling anomalous diffusion," Physical Review E, vol. 63, no. 2, Article ID 021112, 6 pages, 2001.

[5] R. L. Magin, "Fractional calculus in bioengineering," Critical Reviews in Biomedical Engineering, vol. 32, no. 1, pp. 1112-1117, 2004.

[6] R. L. Magin, "Fractional calculus in bioengineering, part 2," Critical Reviews in Biomedical Engineering, vol. 32, no. 2, pp. 105-193, 2004.

[7] R. L. Magin, "Fractional calculus in bioengineering, part 3," Critical Reviews in Biomedical Engineering, vol. 32, no. 3-4, pp. 195-377, 2004.

[8] F. Liu, V. Anh, I. Turner, and P. Zhuang, "Time fractional advection-dispersion equation," Journal of Applied Mathematics \& Computing, vol. 13, no. 1-2, pp. 233-245, 2003.

[9] F. Liu, V. Anh, and I. Turner, "Numerical solution of the space fractional Fokker-Planck equation," Journal of Computational and Applied Mathematics, vol. 166, no. 1, pp. 209-219, 2004.

[10] R. Schumer, D. A. Benson, M. M. Meerschaert, and B. Baeumer, "Multiscaling fractional advectiondispersion equations and their solutions," Water Resources Research, vol. 39, pp. 1022-1032, 2003.

[11] Y. A. Rossikhin and M. V. Shitikova, "Applications of fractional calculus to dynamic problems of linear and nonlinear hereditary mechanics of solids," Applied Mechanics Reviews, vol. 50, no. 1, pp. 15-67, 1997.

[12] T. T. Hartley, C. F. Lorenzo, and H. K. Qammer, "Chaos in a fractional order Chua's system," IEEE Transactions on Circuits and Systems I, vol. 42, no. 8, pp. 485-490, 1995.

[13] I. Petráš, "Chaos in the fractional-order Volta's system: modeling and simulation," Nonlinear Dynamics, vol. 57, no. 1-2, pp. 157-170, 2009.

[14] M.S. Tavazoei and M. Haeri, "Chaotic attractors in incommensurate fractional order systems," Physica D, vol. 237, no. 20, pp. 2628-2637, 2008.

[15] J. T. Machado, “Discrete-time fractional-order controllers," Fractional Calculus \& Applied Analysis, vol. 4, no. 1, pp. 47-66, 2001.

[16] W. Wyss, “The fractional Black-Scholes equation," Fractional Calculus and Applied Analysis, vol. 3, no. 1, pp. 51-61, 2000.

[17] E. Scalas, R. Gorenflo, and F. Mainardi, "Fractional calculus and continuous-time finance," Physica A, vol. 284, no. 1-4, pp. 376-384, 2000.

[18] M. Raberto, E. Scalas, and F. Mainardi, “Waiting-times and returns in high-frequency financial data: an empirical study," Physica A, vol. 314, no. 1-4, pp. 749-755, 2002.

[19] I. Podlubny, Fractional Differential Equations, Academic Press, New York, NY, USA, 1999.

[20] V. Daftardar-Gejji and S. Bhalekar, "Solving multi-term linear and non-linear diffusion-wave equations of fractional order by Adomian decomposition method," Applied Mathematics and Computation, vol. 202, no. 1, pp. 113-120, 2008.

[21] N. H. Sweilam, M. M. Khader, and R. F. Al-Bar, "Numerical studies for a multi-order fractional differential equation," Physics Letters A, vol. 371, no. 1-2, pp. 26-33, 2007.

[22] I. Hashim, O. Abdulaziz, and S. Momani, "Homotopy analysis method for fractional IVPs," Communications in Nonlinear Science and Numerical Simulation, vol. 14, no. 3, pp. 674-684, 2009. 
[23] F. Liu, P. Zhuang, V. Anh, I. Turner, and K. Burrage, "Stability and convergence of the difference methods for the space-time fractional advection-diffusion equation," Applied Mathematics and Computation, vol. 191, no. 1, pp. 12-20, 2007.

[24] E. A. Rawashdeh, "Numerical solution of fractional integro-differential equations by collocation method," Applied Mathematics and Computation, vol. 176, no. 1, pp. 1-6, 2006.

[25] S. Momani and Z. Odibat, "Analytical approach to linear fractional partial differential equations arising in fluid mechanics," Physics Letters A, vol. 355, no. 4-5, pp. 271-279, 2006.

[26] S. Momani and Z. Odibat, "Numerical comparison of methods for solving linear differential equations of fractional order," Chaos, Solitons E Fractals, vol. 31, no. 5, pp. 1248-1255, 2007.

[27] Z. Odibat and S. Momani, "Application of variational iteration method to nonlinear differential equations of fractional order," International Journal of Nonlinear Sciences and Numerical Simulation, vol. 7, no. 1, pp. 27-34, 2006.

[28] W. Jiang and Y. Z. Lin, "Approximate solution of the fractional advection-dispersion equation," Computer Physics Communications, vol. 181, no. 3, pp. 557-561, 2010.

[29] R. Pandey, O. P. Singh, and V. Baranwal, "An analytic algorithm for the space-time fractional advection-dispersion equation," Computer Physics Communications, vol. 182, no. 5, pp. 1134-1144, 2011.

[30] S. Badraoui, "Approximate controllability of a reaction-diffusion system with a cross-diffusion matrix and fractional derivatives on bounded domains," Boundary Value Problems, vol. 2010, pp. 1-14, 2010.

[31] O. Zaid and S. Momani, "The variational iteration method: an efficient scheme for handling fractional partial differential equations in fluid mechanics," Computers $\mathcal{E}$ Mathematics with Applications, vol. 58, no. 11-12, pp. 2199-2208, 2009.

[32] G. J. Fix and J. P. Roop, "Least squares finite-element solution of a fractional order two-point boundary value problem," Computers \& Mathematics with Applications, vol. 48, no. 7-8, pp. 1017-1033, 2004.

[33] B. Ahmad and G. Wang, "A study of an impulsive four-point nonlocal boundary value problem of nonlinear fractional differential equations," Computers \& Mathematics with Applications, vol. 62, no. 3, pp. 1341-1349, 2011.

[34] M. Rehman and R. Khan, "Existence and uniqueness of solutions for multi-point boundary value problems for fractional differential equations," Applied Mathematics Letters, vol. 23, no. 9, pp. 10381044, 2010.

[35] S. Liang and J. Zhang, "Existence and uniqueness of strictly nondecreasing and positive solution for a fractional three-point boundary value problem," Computers \& Mathematics with Applications, vol. 62, no. 3, p. 1333-1340, 2011.

[36] Z. Chen and Y. Z. Lin, "The exact solution of a linear integral equation with weakly singular kernel," Journal of Mathematical Analysis and Applications, vol. 344, no. 2, pp. 726-734, 2008.

[37] Y. Z. Lin, J. Niu, and M. G. Cui, “A numerical solution to nonlinear second order three-point boundary value problems in the reproducing kernel space," Applied Mathematics and Computation, vol. 218, no. 14, pp. 7362-7368, 2012.

[38] J. Niu, Y. Z. Lin, and C. P. Zhang, "Approximate solution of nonlinear multi-point boundary value problem on the half-line," Mathematical Modelling and Analysis, vol. 17, no. 2, pp. 190-202, 2012.

[39] K. Miller and B. Ross, An Introduction to the Fractional Calculus and Fractional Differential Equations, John Wiley \& Sons, New York, NY, USA, 1993.

[40] M. G. Cui and Y. Z. Lin, Nonlinear Numerical Analysis in the Reproducing Kernel Space, Nova Science, New York, NY, USA, 2009.

[41] S. Momani and M. Noor, "Numerical methods for fourth-order fractional integro-differential equations," Applied Mathematics and Computation, vol. 182, no. 1, pp. 754-760, 2006. 


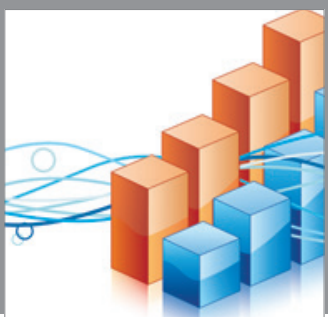

Advances in

Operations Research

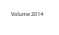

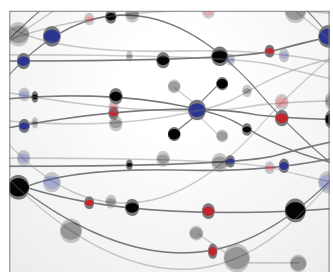

\section{The Scientific} World Journal
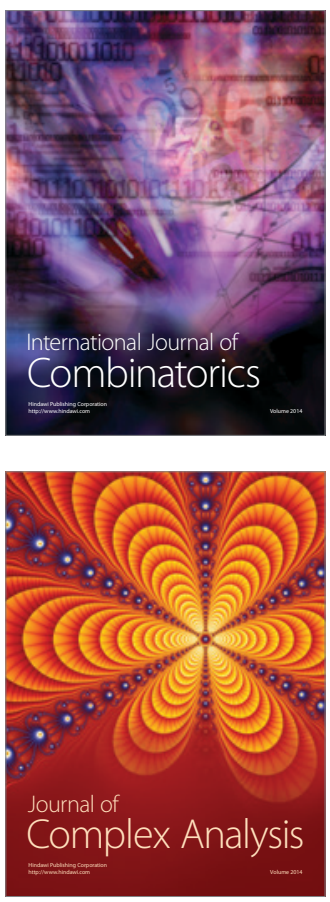

International Journal of

Mathematics and

Mathematical

Sciences
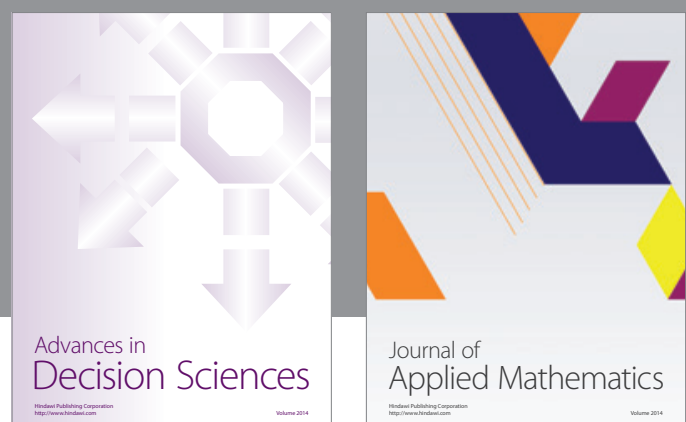

Journal of

Applied Mathematics
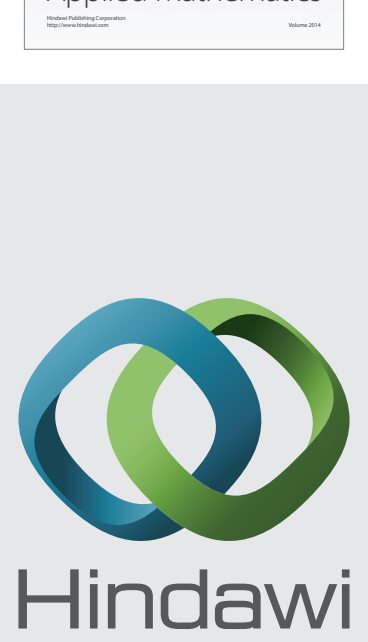

Submit your manuscripts at http://www.hindawi.com
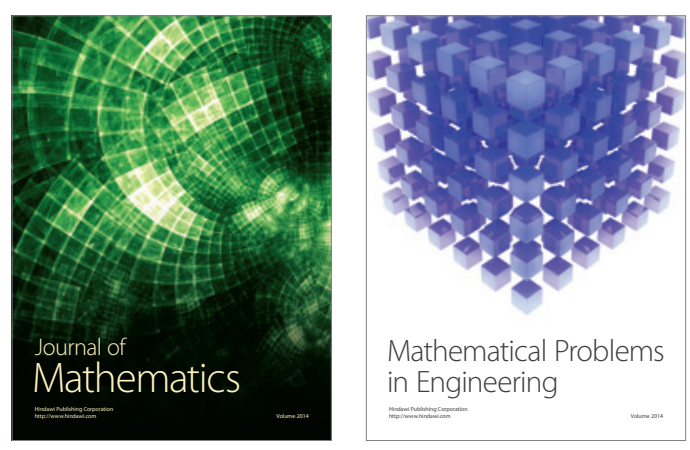

Mathematical Problems in Engineering
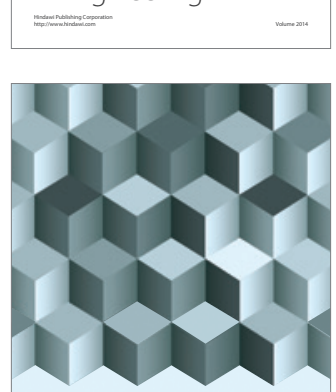

Journal of

Function Spaces
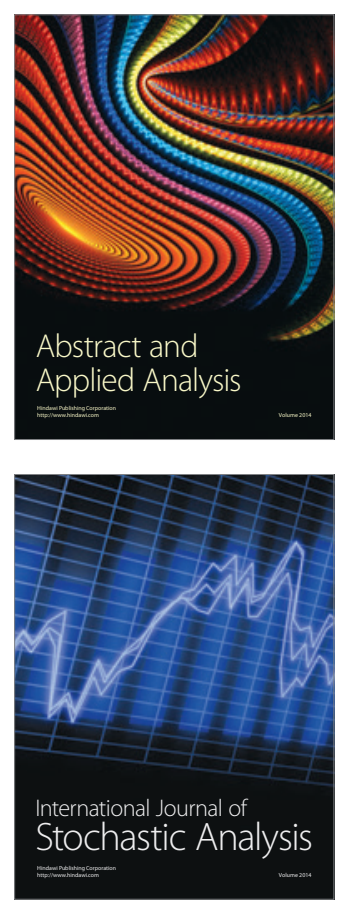

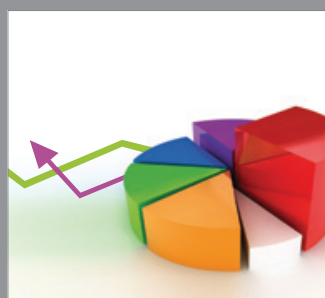

ournal of

Probability and Statistics

Promensencen
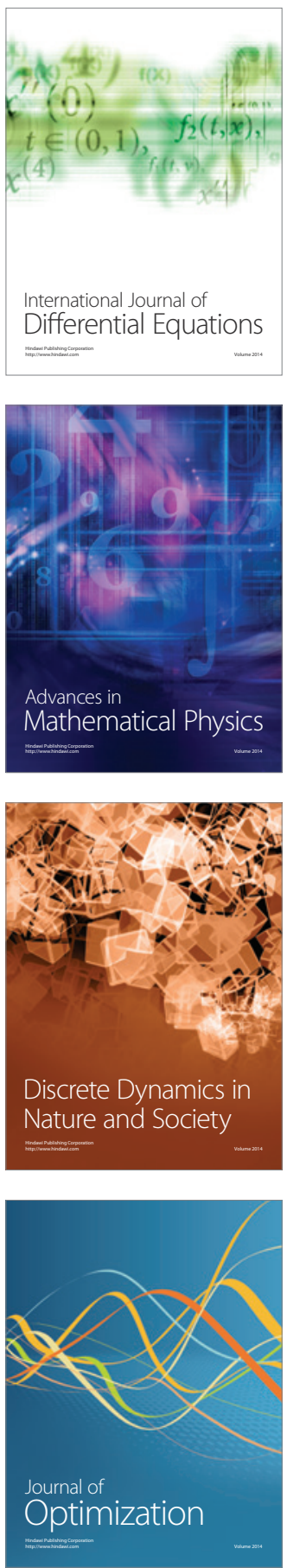